\title{
A Rare Case of Osteoinvasive Amelanotic Melanoma of the Nail Unit
}

\author{
Alexandra T. Black ${ }^{a}$ Arash H. Lahouti ${ }^{b}$ Iskender S. Genco ${ }^{c}$ \\ Matvey Yagudayev $^{\mathrm{a}}$ Bryan C. Markinson $^{d}$ William D. Spielfogel ${ }^{\mathrm{a}}$ \\ aDepartment of Orthopedics, Lenox Hill Hospital Northwell, New York, NY, USA; b Department of Pathology, \\ Lenox Hill Hospital Northwell, New York, NY, USA; 'Department of Pathology and Laboratory Medicine,

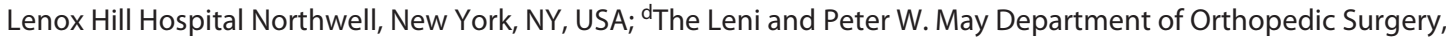 \\ Icahn School of Medicine at Mount Sinai, New York, NY, USA
}

\section{Established Facts}

- Amelanotic melanoma is a rare subtype.

- Clinically difficult to diagnose due to lack of pigmentation and variable histopathological features.

- Amelanotic lesions are known to mimic a wide differential of diagnoses.

\section{Novel Insights}

- Amelanotic variants of the nail unit with osseous involvement are rare with few reported cases in the literature.

- In cases of osteoinvasion, magnetic resonance imaging may detect abnormal bone marrow signal changes, while plain film radiographs are negative, suggestive of inflammation similar in appearance to osteomyelitis.

- A heightened index of suspicion should be practiced with a low threshold to biopsy suspicious, nonhealing lesions in order to establish a diagnosis at the earliest possible moment.

\section{Keywords}

Melanoma · Amelanotic melanoma · Biopsy · Nail unit ·

Osteoinvasion · Tumor · Surgery · Dermatopathology

\section{Abstract \\ Introduction: Amelanotic melanoma is a rare subtype, which may be clinically difficult to diagnose due to lack of pigmenta- tion and variable histopathological features. Osteoinvasion is another rare characteristic of melanoma. There are few re-}

ports in the literature of amelanotic melanoma of the nail unit (nail bed, matrix, and nail folds) with invasion of bone. Case Presentation: We present a case of a 73-year-old Caucasian male with a 13-month history of an ungual lesion on his right hallux. The lesion was initially treated as a chronic diabetic ulceration with failure to resolve with standard of care. Discussion/Conclusion: A heightened index of suspicion for a malignant process is necessary when standard of care fails to lead to improvement or resolution. In these instances, biopsy should be seriously considered.

(c) 2020 S. Karger AG, Basel

$\begin{aligned} & \text { karger@karger.com } \\ & \text { www.karger.com/sad }\end{aligned}$
Karger ${ }^{\prime /}$




\section{Introduction}

According to the American Cancer Society, in 2020, the estimated number of new cases of melanoma in the USA is 100,350 and the estimated number of related deaths is 6,850 [1]. Nail unit melanoma represents approximately $0.7-8 \%$ of all melanoma cases $[1,2]$. About $15-25 \%$ of nail unit melanoma are amelanotic variants [1-3]. A large percentage, $>90 \%$ of nail unit melanoma, occur in the thumb or the hallux $[4,5]$. Amelanotic melanoma has been found among all melanoma subtypes: superficial spreading, nodular, lentigo maligna, and acral lentiginous melanoma [4]. Upon clinical examination, these lesions may entirely or partially lack pigmentation. Lack of pigmentation clinically poses a challenge for accurate diagnosis because these lesions are known to mimic a wide differential of diagnoses. Notably, osseous involvement is an uncommon feature of melanoma. We present a case of an unusual presentation of amelanotic melanoma of the nail unit involving penetration of the underlying bone as confirmed by histopathological analysis.

\section{Case Report/Case Presentation}

A 73-year-old Caucasian male with a past medial history including diabetes mellitus type II, hyperlipidemia, hypertension, coronary artery disease, peripheral vascular disease, and gastroesophageal reflux presented with a 13-month history of a lesion on his right hallux. The patient initially received treatment for a nonhealing lesion with wound care and antibiotics at a different facility with a diagnosis of a diabetic foot infection. The patient reported that the lesion progressively grew in size. It was ultimately referred to our service for management of this lesion.

On examination, a granulomatous, hypervascular lesion measuring $4 \times 4 \mathrm{~mm}$, totally consuming the nail bed, was noted. There was no nail plate present (shown in Fig. 1). The lesion bled upon gentle manipulation and probing. AP, lateral, and oblique radiograph views of the right foot were negative for cortical erosions and periosteal reaction. Magnetic resonance imaging (MRI) revealed abnormal bone marrow signal, causing concern for osteomyelitis.

Based on the history and imaging findings, the decision was made to sample the lesion. A biopsy into the bulk of the lesion was performed and a bone specimen was procured percutaneously through a separate site. The bone quality was normal, while the soft tissue specimen was spongy and soft. Histologic analysis did not reveal the presence of an infectious process or necrotic tissue, even though microbiology was positive for Enterococcus faecalis, which was considered a contaminant. Biopsy results revealed malignant melanoma of the right great toe. Tumor cells were positive for HMB45, s100, and Melan-A and negative for cytokeratin, supporting the diagnosis of melanoma.

The patient was referred to a surgical oncologist for staging. Lymphadenopathy of the inguinal region was appreciated on ini-

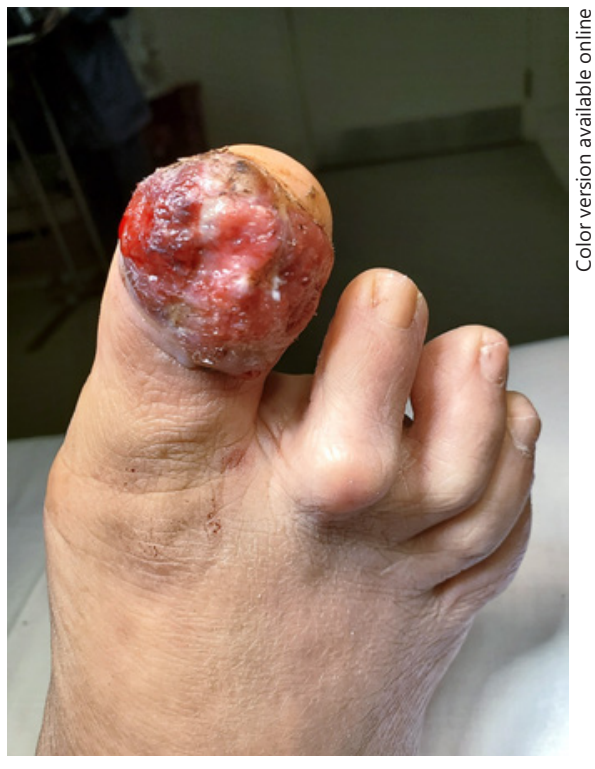

Fig. 1. Clinical photo demonstrating a hypervascular, nonpigmented lesion on the dorsum right distal hallux.

tial examination. On positron emission tomography/computed tomography imaging, a $2.2 \times 2.1-\mathrm{cm}$ inguinal/femoral lymph node demonstrated increased uptake. Chest and abdomen positron emission tomography/computed tomography imaging were negative. An ultrasound-guided core needle biopsy of the lymph node specimen was obtained and sent for pathologic analysis, revealing melanoma. Based on these findings, local wide excision was performed resulting in a right hallux amputation. Simultaneously, the vascular and surgical oncology teams performed right groin superficial lymph node dissection with a sartorius muscle flap to protect the femoral vessels after nuclear medicine lymphoscintigraphy demonstrated activity within 3 foci in the medial right inguinal region.

Gross examination of the specimen showed a 4.4 -cm distal portion of the hallux. On sectioning, a $3.8 \times 3.4-\mathrm{cm}$ mass with a tan, friable cut surface was identified. The mass appeared to invade the underlying bone. Histologic examination demonstrated invasive melanoma of the right hallux invading subcutaneous tissue and bone, measuring $3.8 \mathrm{~cm}$ in greatest dimension (Fig. 2-4). Furthermore, margins were negative for tumor with a maximum tumor thickness of $9 \mathrm{~mm}$, a mitotic rate 13 mitoses $/ \mathrm{mm}^{2}$, and a Clark level of V. From the radical groin dissection, 1 out of 6 lymph nodes were positive for tumor. The largest metastatic deposit measured $6 \mathrm{~cm}$.

\section{Discussion/Conclusion}

We present an unusual case of amelanotic melanoma where the primary lesion was located in the nail unit with invasion of underlying bone and a positive unilateral inguinal lymph node (pT4 pN1b). Our patient presented 


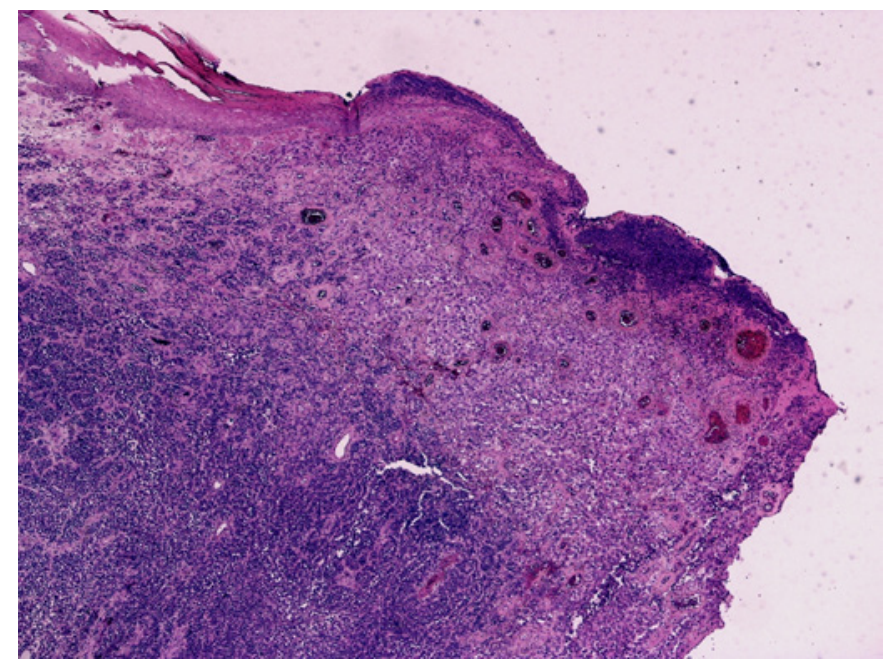

Fig. 2. $\mathrm{H}$ and $\mathrm{E}$ section showing ulceration of the epidermis with a solid infiltrative growth.

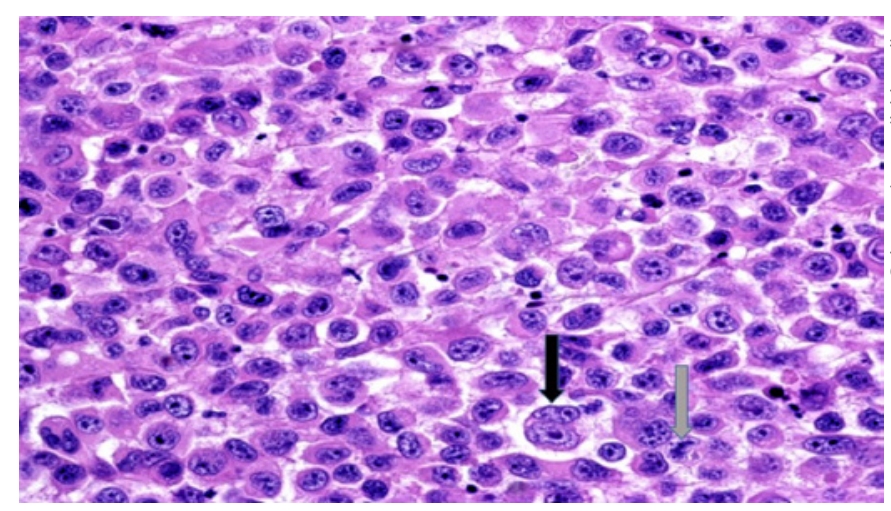

Fig. 3. $\mathrm{H}$ and $\mathrm{E}$ section showing eosinophilic cytoplasm, pleomorphic tumor cells, and prominent nucleoli with binucleation cells (black arrow) and multiple mitoses (gray arrow).

with a suspicious-looking nail unit lesion with the absence of a nail plate. With a past medical history consisting of diabetes mellitus, the patient was initially treated for a diabetic foot ulcer prior to presentation. Infections are common in the setting of lower extremity pathology and, for any presumed infectious process that does not respond to conventional medical management, the underlying etiology should be reconsidered [6].

Malignant melanoma invades by local extension by the lymphatic system. Direct invasion of bone is an unknown process. In general, osseous invasion usually occurs in patients in later stages of the disease process, presenting with widespread metastases [7]. Malignant melanoma is

Osteoinvasive Amelanotic Melanoma of the Nail Unit

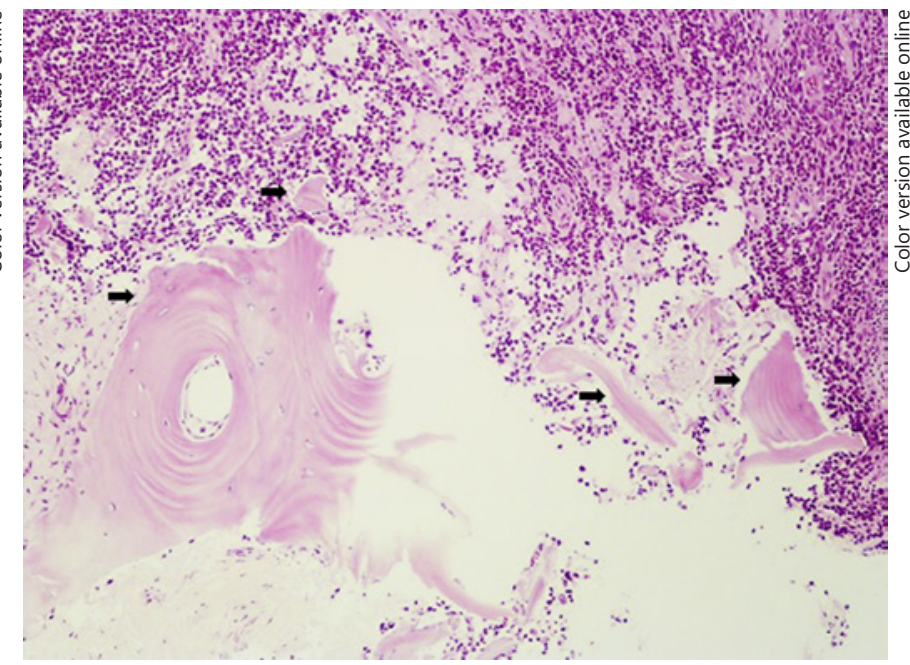

Fig. 4. Representative picture of osteoinvasion. Tumor cells (upper) infiltrating and destructing the underlying osseous tissue (arrows).

more likely to spread to the liver and brain compared to bone metastases [8]. To the authors' knowledge, there are 2 reported cases of osteoinvasive amelanotic melanoma involving the subungual region of the hallux, including the present study. Gooselink et al. [9] presents a case where a subungual amelanotic lesion of the hallux invasive to bone presented with a vascularized, noncompressible lesion revealing no signs of infection with erosions and "increased soft tissue density in a starburst pattern with soft tissue calcifications" on plain film radiographs. The preoperative plain films obtained for the present study were negative for pathologic changes on plain film imaging; however, MRI revealed bone marrow signal changes suggestive of possible osteomyelitis. Although nonspecific, this MRI read detected changes in the bone related to the osteoinvasive nature of the tumor.

Amelanotic melanoma is a rare form of skin cancer and clinically difficult to diagnose due to lack of pigmentation and variable histopathological features. Melanocytic tumor cells usually are deficient in producing melanoma but may do so in varying amounts. In our patient, histologic features included ulceration of the epidermis with a solid infiltrative growth pattern and invasion of bone (Fig. 4). On higher power, pleomorphic cells, prominent nucleoli, binucleated cells, multiple mitoses, and vesicular nuclei were appreciated (Fig. 2, 3). On physical examination, amelanotic lesions are usually red, pink or flesh-colored, friable, and hypervascular with a tendency to bleed. Bleeding may indicate ulceration, which is a poor prognostic factor. Thickness is another important

Skin Appendage Disord 2021;7:139-143 141 
prognostic indicator; however, dermal invasion may be overestimated due to variation in epidermal thickness of different areas of the nail unit in addition to epidermal hyperplasia [10]. Amelanotic variants and melanoma of the nail unit do not display typical ABCDE criteria, which is classically utilized for clinical evaluation of pigmented melanoma. Amelanotic melanoma tends to present at more advanced stages compared to pigmented lesions and are associated with higher AJCC tumor staging at the time of diagnosis with a higher mortality [4]. These unusual features associated with amelanotic variants and nail unit melanoma may cause a delay in treatment with worse prognostic outcomes.

Delays in diagnosis of amelanotic acral lesions, particularly involving the nail unit, is a frequent issue secondary to inconsistent features of early melanoma potentially leading to diagnosis in later stages of development. Two main patterns of subungual melanoma have been described: longitudinal melanonychia and amelanotic tumors [11]. Longitudinal melanonychia originates from the nail matrix, while nonpigmented subungual nodules usually coupled with onycholysis typically originate from the nail bed [12]. Dermal invasion of subungual melanoma occurs at later stages, and this might be explained by radial growth typically seen earlier on $[13,14]$. Shin et al. [15] studied 23 subungual melanoma (5 in situ melanoma and 18 invasive melanoma) cases involving the nail matrix as well as other parts of the nail unit and examined the invasion pattern and thickness on longitudinal histologic sections. Among the 18 invasive melanoma cases, the nail matrix showed either in situ melanoma or thinner dermal invasion compared to other parts of the nail unit. They concluded that the nail matrix onychodermis appears to be more resistant to invasion which may explain the rare occurrence of osteoinvasion in subungual melanoma despite the close proximity between the nail matrix and underlying bone [13].

Non-amputative surgery may be possible for subungual melanoma with smaller thickness or in earlier stages [15]. On the other hand, the nail matrix is relatively thin and close to the phalanx. This anatomical relationship might encourage surgeons to opt for amputation as opposed to less aggressive surgical management [16]. Bristow et al. [11] suggest a specialist referral and surgical removal for histologic purposes when presented with a pyogenic granuloma, granulomatous masses, lesions that do not respond within 2 months, and any lesions that disturb the integrity of the nail. A heightened index of suspicion is necessary in any nonhealing and suspiciouslooking lesion. These amelanotic nail unit lesions with osseous involvement are uncommon or underreported in the literature, and further research is warranted to grasp a better understanding of this entity. Clinicians should practice maintaining a low threshold to biopsy suspicious, nonhealing lesions.

\section{Statement of Ethics}

This research was conducted ethically in accordance with the World Medical Association Declaration of Helsinki. Patient information was de-identified in this report. Written informed consent was obtained from the patient for publication of this case report and any accompanying images.

\section{Conflict of Interest Statement}

The authors have no conflicts of interest to declare.

\section{Funding Sources}

There is no funding of any research relevant to this study.

\section{Author Contributions}

M.Y. and W.D.S. conceived the presented idea and were involved in the treatment of the patient presented. A.T.B. wrote the manuscript with support from B.C.M., M.Y., and W.D.S. A.H.L. and I.S.G. are responsible for the photomicrographs and histopathological analysis.

\section{References}

1 American cancer society [Internet]. Cancer statistics: A Cancer Journal for Clinicians [cited 2020 Mar 5]. Available from: https://acsjournals.onlinelibrary.wiley.com/doi/ full/10.3322/caac. 21590 .

2 Levit EK, Kagen MH, Scher RK, Grossman M, Altman E. The ABC rule for clinical detection of subungual melanoma. J Am Acad Dermatol. 2000;42(2 Pt 1):269-74.
3 Riahi RR, Cohen PR, Goldberg LH. Subungual amelanotic melanoma masquerading as onychomycosis. Cureus. 2018;10(3):e2307.

4 Thomas NE, Kricker A, Waxweiler WT, Dillon PM, Busman KJ, From L, et al. Comparison of clinicopathologic features and survival of histopathologically amelanotic and pigmented melanomas: a population-based study. JAMA Dermatol. 2014;150(12):1306-314.
5 Arican O, Sasmaz S, Coban YK, Ciralik H. Subungual amelanotic malignant melanoma. Saudi Med J. 2009;27(2):247-9.

6 Markinson BC, Stowers JM, Black A, Saccomanno R, Desman G. The misdiagnosis of acral lentiginous melanoma: three case presentations. J Am Podiatr Med Assoc. 2019; 109(2):166-171. 
7 Gómez-León N, Pacheco-Barcia V, Ballesteros AI, Fraga J, Colomer R, Friera A. Skeletal muscle and solitary bone metastases from malignant melanoma: multimodality imaging and oncological outcome. Melanoma Res. 2018;28(6):562-70

8 Lee YT. Malignant melanoma: pattern of metastasis. CA Cancer J Clin. 1980;30(3):137-42.

9 Gosselink CP, Sindone JL, Meadows BJ, Mohammadi A, Rosa M. Amelanotic subungual melanoma: a case report. J Foot Ankle Surg. 2009;48(2):220-4.

10 Tan KB, Moncrieff M, Thompson JF, McCarthy SW, Shaw HM, Quinn MJ, et al. Subungual melanoma: a study of 124 cases highlighting features of early lesions, potential pitfalls in diagnosis, and guidelines for histologic reporting. Am J Surg Pathol. 2007;31(12):1902-12.
11 Bristow IR, de Berker DA, Acland KM, Turner RJ, Bowling J. Clinical guidelines for the recognition of melanoma of the foot and nail unit. J Foot Ankle Res. 2010;3:25.

12 Tosti A, Richert B, Pazzaglia M. Tumors of the nail apparatus. In: Scher RK, Daniel CR, editors. Nails: diagnosis, therapy, surgery. 3rd ed. Oxford, UK: Elsevier Saunders; 2005. p. 202.

13 Haneke E. Surgical anatomy of the nail apparatus. In: Richert B, Di Chiacchio N, Haneke E, editors. Nail surgery. New York, NY, USA: Informa Healthcare; 2011. Vol. 1.
14 Izumi M, Ohara K, Hoashi T, Nakayama H, Chiu CS, Nagai T, et al. Subungual melanoma: histological examination of 50 cases from early stage to bone invasion. J Dermatol. 2008; 35(11):695-703.

15 Shin HT, Jang KT, Mun GH, Lee DY, Lee JB. Histopathological analysis of the progression pattern of subungual melanoma: late tendency of dermal invasion in the nail matrix area. Mod Pathol. 2014;27(11):1461-7.

16 Kim JY, Jung HJ, Lee WJ, Kim DW, Yoon GS, Kim DS, et al. Is the distance enough to eradicate in situ or early invasive subungual melanoma by wide local excision? From the point of view of matrix-to-bone distance for safe inferior surgical margin in Koreans. Dermatology. 2011;223(2):122-3. 